\title{
Combination treatment with metronomic temozolomide, bevacizumab and long-acting octreotide for malignant neuroendocrine tumours
}

\section{Dear Editor}

Neuroendocrine tumours (NETs) are highly vascularised tumours that express high levels of the vascular endothelial growth factor (VEGF) ligand together with its receptor VEGFR (Modlin et al. 2008). Although advanced NETs may exhibit a $30-40 \%$ response rate to combination chemotherapeutic approaches, the response to single-agent chemotherapy is only $10 \%$ (Modlin et al. 2008). Bevacizumab (BVZ; Avastin, Roche, Basle, Switzerland), an anti-VEGF humanised monoclonal antibody, has been shown to exert objective tumour responses and improvement in median time to progression (TTP) in advanced carcinoid tumours (Yao et al. 2008). Additionally, a recently published study reported that temozolomide (TMZ), an oral chemotherapy derivative of dacarbazine, at a dose of $200 \mathrm{mg} / \mathrm{m}^{2}$ on first 5 days of each 28-day cycle may exert a significant effect on NETs (Ekeblad et al. 2007). A previous report that examined a variety of NETs suggested that the combination of BVZ and TMZ can be safely administered and shows promising activity in patients who had failed to prior treatments (Kulke et al. 2006a). Additionally, there is growing interest on regimens that introduce continuous low-dose TMZ administration as protracted low-dose TMZ regimens may deplete O6-methylguanine DNA methyltransferase (MGMT), an important factor in cases of TMZ resistance and/or inhibit endothelial cell proliferation and formation of tumour vasculature via the so-called metronomic effect (Tolcher et al. 2003, Lam et al. 2007). Although the exact mechanism of action of somatostatin analogues is not well understood, a long-standing hypothesis based on preclinical experiments suggests that they exert an antiangiogenic effect (Grozinsky-Glasberg et al. 2008).

Given the high degree of endothelial proliferation, high vascular permeability and high expression of proangiogenic growth factors such as VEGF in NETs, angiogenesis inhibition by multiple pathways may be a rational treatment strategy for these tumours. We conducted a pilot study to assess the toxicity and efficacy of a combination antiangiogenic regimen including a metronomic TMZ schedule, BVZ and octreotide in a cohort of patients with a variety of advanced NETs that had progressed on previous therapies.

From January 2007 until January 2009, 15 patients with advanced NETs, mainly grade II tumours with Ki-67 labelling index (LI) 3-19\%, were treated with combination treatment including oral TMZ (Temodal, MSD, Geneva, Switzerland) at a continuous standard daily dose of $100 \mathrm{mg}$ before bedtime, BVZ $7.5 \mathrm{mg} / \mathrm{m}^{2}$ every 3 weeks i.v. and $30 \mathrm{mg}$ of the somatostatin analogue, octreotide long-acting release (Sandostatin $30 \mathrm{mg}$ LAR; Novartis, Basle, Switzerland) i.m. every 4 weeks. The study was approved by the Institutional Review Board and Ethics Committee at each participating centre and was conducted in accordance with Good Clinical Practice principles and applicable local regulations. All patients provided written informed consent. The cycle duration was considered to be 3 weeks based on the BVZ infusion. After a median of 18 months of follow-up, data were analysed for toxicity, chromogranin $\mathrm{A}(\mathrm{CgA})$ percentage variations, best radiological response and TTP. Adverse events were evaluated according to the Common Toxicity Criteria, version 3.0 of the National Cancer Institute. CgA (normal value 12-98 $\mathrm{ng} / \mathrm{ml}$ ) levels were evaluated before the first cycle and every two cycles, whereas imaging with triple-phase computed tomography or magnetic resonance imaging was performed every four cycles. Radiological response was classified according to Response Evaluation Criteria in Solid Tumours version 1.1 (Eisenhauer et al. 2009). TTP was defined as the time from treatment initiation until radiological tumour progression. The extent of disease, based on the American Joint Committee on Cancer Staging Manual, 7th edition, and the grading of the tumours based on the recent WHO classification (Bosman et al. 2010) were recorded for each patient and assessed centrally by the same radiologist and pathologist with a special interest in NETs.

DOI: $10.1530 / E R C-11-0287$ 
Fifteen patients, with advanced NETs, who progressed after at least one regimen of chemotherapy, were enrolled over a 2-year period in two participating centres in this study of a combined treatment schedule including TMZ, BVZ and a somatostatin analogue (temozolomide, bevacizumab \& somatostatin analogue; TeBeSa). Patients had a median age of 62.5 (range 47-78) years and received a median number of 12 (range 4-20) cycles. Patient characteristics including demographics and previous treatments are shown in Table 1.

Toxicity was observed in six out of $15(40 \%)$ patients and included one patient with Grade III vomiting $2 \mathrm{~h}$ following ingestion of the first TMZ capsule regardless of the co-administration of metoclopramide $20 \mathrm{mg}$ orally. Two patients developed grade I neutropenia that required discontinuation of TMZ capsules for 1 week. One patient developed Grade II thrombocytopenia, and TMZ treatment was discontinued and restarted 3 weeks after the platelet count recovered. Two patients developed mild hypertension, most probably related to BVZ that was treated with oral antihypertensives. There were no side effects related to the use of the somatostatin analogue.

All patients had elevated $\mathrm{CgA}$ levels before initiation of treatment. Of the 14 evaluable patients, $57 \%(8 / 14)$ had a $50-100 \%$ drop, $35 \%(5 / 14)$ had a $25-50 \%$ drop or $<25 \%$ increase and $7 \%(1 / 14)$ had increase in $\mathrm{CgA}$ levels.

Radiological responses included one (7\%) complete response (CR), eight (57\%) partial responses (PRs), three $(21 \%)$ stable diseases (SDs) and two (14\%) progressive diseases (PDs). Fifty-seven per cent (8/14) of patients achieved a PR after a median of 12.5 (range 8-20) cycles. Two of these patients completed 12 and 16 cycles, respectively, and their best radiological responses are shown in Fig. 1. Three $(21.5 \%)$ patients had prolonged periods of SD; no radiological change was detected over a median of 12.6 (range 11-15) cycles. Two (14\%) patients had PD after the fourth and sixth cycles and discontinued their treatment. In evaluable patients, the median TTP was 36 weeks (range 10-60 weeks, mean 33.50, s.D. 14.37 , 95\% confidence interval $=25.2-41.8$ ). In our cohort of patients, only five had tumour with Ki-67 $>6 \%$. Of these, one had PD (lung), three had SD and only one had a PR as described in the first case above.

During the past 4 years, there have been six reports showing activity of TMZ in patients with advanced NETs. These included a monotherapy protocol (Ekeblad et al. 2007), three different combinations of TMZ with BVZ (Kulke et al. 2006a), thalidomide (Kulke et al. 2006b) and everolimus (Kulke et al. 2010)
Table 1 Patient characteristics and previous treatments

\begin{tabular}{lc}
\hline Patient characteristics & $\boldsymbol{n}(\%)$ \\
\hline Median age (range) & $47-78(62.5)$ years \\
Male & $67(10)$ \\
Female & $33(5)$ \\
Ki-67: 6-19\% & $33(5)$ \\
Ki-67: 2-5\% & $67(10)$ \\
Functioning NETs & $27(4)$ \\
Non-functioning NETs & $73(11)$ \\
Site of origin & \\
Pancreas & $47(7)$ \\
Small intestine & $20(3)$ \\
Duodenum & $1(7)$ \\
Stomach & $1(7)$ \\
Rectum & $1(7)$ \\
Lung & $1(7)$ \\
Unknown & $1(7)$ \\
Stage (AJCC 7th edn) & \\
IIIA and B & $1(7)$ \\
IV (Liver) & $80(12)$ \\
IV (other distant organs) & $13(2)$ \\
Treatments before initiation of & \\
TeBeSa & $15(100)$ \\
Surgery & $1(7)$ \\
Debulking & \\
Whipple & $67(10)$ \\
Radiolabelled peptides & $28(4)$ \\
Chemotherapy & $1(7)$ \\
Platinum based & \\
Streptozotocin/5-fluorouracil & \\
Anthracycline & $13(2)$ \\
Docetaxel & $80(12)$ \\
Other medical therapies & $53(8)$ \\
Interferon & $40(6)$ \\
Octreotide long-acting release \\
Everolimus \\
Lines of medical therapies \\
1 (5) \\
2 \\
3 \\
\hline
\end{tabular}

and two reports investigating the combination of TMZ with capecitabine (Strosberg et al. 2011, Welin et al. 2011). However, previous protocols differ from that used in this study in the TMZ administration schedule, as in all of these studies, TMZ was administered at a higher dose for fewer days (Kulke et al. 2006a,b, 2010, Ekeblad et al. 2007, Strosberg et al. 2011, Welin et al. 2011). Our study, although sample size is small, supports the use of a continuous daily dose of $100 \mathrm{mg}$ TMZ as the overall response rate (PR and CR rate) was $64 \%$, compared with previously reported that ranged between 16 and 45\% (Kulke et al. 2006a,b, 2010). There have been two studies testing TMZ monotherapy or in combination with capecitabine in advanced NETs reporting different response rates (70 and 33\%; Strosberg et al. 2011, Welin et al. 2011). This difference could be attributed to the different 
(a)

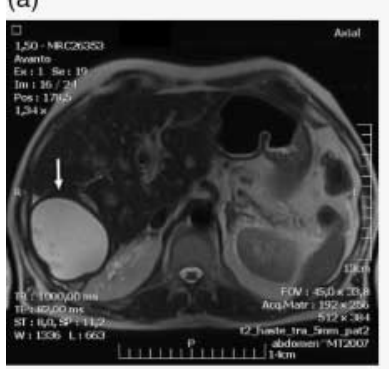

(b)

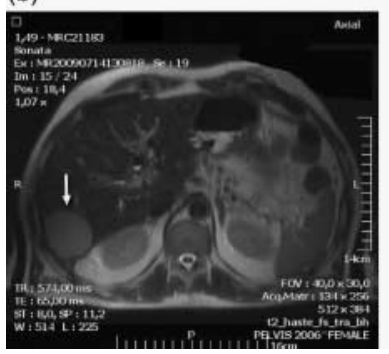

(c)

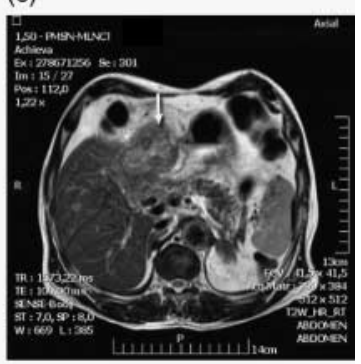

(d)

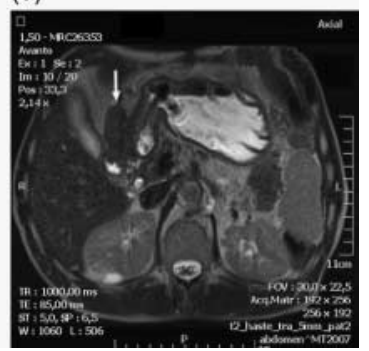

Figure 1 Downstaging of gastroenteropancreatic tumours (arrows) after TeBeSa combination therapy. MRI details shown before treatment (a) and (c) and after six cycles (b) and (d) for two patients with a pancreatic (a)/(b) and a duodenal tumour $(\mathrm{c}) /(\mathrm{d})$.

histological groups of patients included in the two studies as well as to the fact that in the first study patients were chemotherapy naive. In our study, $80 \%$ of patients were previously treated with chemotherapy. Although we did not examine MGMT expression, the high percentage of responses observed is in accordance with an earlier observation, suggesting that protracted administration of TMZ even leads to significant and prolonged depletion of MGMT activity (Tolcher et al. 2003). However, a recent retrospective study on patients with advanced NETs receiving TMZ suggested that MGMT methylation status did not correlate with clinical responses (Welin et al. 2011). In our small cohort of patients, therapeutic responses were accompanied by a grade III vomiting and mild myelosuppression that were manageable compared to previous studies, which reported grade III-IV myelosuppression (Kulke et al. 2006b, Ekeblad et al. 2007).

In studies thus far, the majority of responses to chemotherapy, including TMZ, have been observed in pancreatic NETs (Kulke et al. 2006a,b, Ekeblad et al. 2007, Modlin et al. 2008, Strosberg et al. 2011, Welin et al. 2011). Although our study has limitations such as the heterogeneity of tumour subtypes and the small number of patients included (Kulke et al. 2011), it supports the investigation of metronomic TMZ chemotherapy in pancreatic and non-pancreatic NETs. It has previously been suggested that NETs with $\mathrm{Ki}-67 \mathrm{LI}$ more than $10 \%$ maybe responsive to chemotherapy at an earlier line compared with NETs with $\mathrm{Ki}-67 \mathrm{LI}<5 \%$ that are preferably treated with biological therapies (Oberg 2002). Although our study is small, it showed that tumours with Ki-67 LI 2-5 and 6-19\% have similar response rates to TeBeSa therapy, and overall, this is one of the highest reported rates of single-agent cytotoxics in the literature.

In conclusion, our study suggests that a protracted daily low dose of TMZ together with BVZ and octreotide LAR has activity in advanced, grade II NETs. It is clinically important to explore this combination in the context of large phase II-III studies, including translational studies, investigating the molecular basis of the observed responses and possible biomarkers.

Anna Koumarianou ${ }^{1}$ Stavroula Antoniou ${ }^{2}$ George Kanakis ${ }^{2}$

Nikolaos Economopoulos ${ }^{3}$ Dimitra Rontogianni ${ }^{4}$ Anastasios Ntavatzikos ${ }^{5}$ Nikolaos Tsavaris ${ }^{6}$ Dimitrios Pectasides ${ }^{1}$ George Dimitriadis ${ }^{1}$ Gregory Kaltsas ${ }^{2}$

${ }^{1}$ Medical Oncology Unit,

Second Department of Internal Medicine Propaedeutic, Attikon University General Hospital, Rimini 1, 12462 Athens, Greece

${ }^{2}$ Endocrine Unit, Department of Pathophysiology, Laiko University Hospital, National University of Athens, Mikras Asias 75,

Goudi 11527 Athens, Greece ${ }^{3}$ MRI Unit, Radiology Department, Attikon University General Hospital, Rimini 1, 12462 Athens, Greece

${ }^{4}$ Pathology Department Evangelismos Hospital ${ }^{5}$ Fourth Department of Surgery, Attikon University Hospital and

${ }^{6}$ Medical Oncology Unit, Department of Pathophysiology, Laiko University Hospital, National University of Athens, Mikras Asias 75,

Goudi 11527 Athens, Greece

(Correspondence should be addressed to A Koumarianou; Email: akoumari@yahoo.com) 


\section{Declaration of interest}

The authors declare that there is no conflict of interest that could be perceived as prejudicing the impartiality of the research reported.

\section{Funding}

We thank the Hellenic Anticancer Society for an unrestricted grant on the study of angiogenesis in gastrointestinal tumours.

\section{References}

Bosman F, Carneiro F, Hruban R \& Theise N Eds 2010 In WHO Classification of Tumours of the Digestive System. Lyon, France: IARC Press.

Eisenhauer EA, Therasse P, Bogaerts J, Schwartz LH, Sargent D, Ford R, Dancey J, Arbuck S, Gwyther S, Mooney M et al. 2009 New response evaluation criteria in solid tumours: revised RECIST guideline (version 1.1). European Journal of Cancer 45 228-247. (doi:10.1016/j. ejca.2008.10.026)

Ekeblad S, Sundin A, Janson ET, Welin S, Granberg D, Kindmark H, Dunder K, Kozlovacki G, Orlefors H, Sigurd M et al. 2007 Temozolomide as monotherapy is effective in treatment of advanced malignant neuroendocrine tumors. Clinical Cancer Research 13 2986-2991. (doi:10.1158/1078-0432.CCR-06-2053)

Grozinsky-Glasberg S, Shimon I, Korbonits M \& Grossman AB 2008 Somatostatin analogues in the control of neuroendocrine tumours: efficacy and mechanisms. Endocrine-Related Cancer 15 701-720. (doi:10.1677/ ERC-07-0288)

Kulke M, Stuart K, Earle C, Bhargava P, Clark J, Enzinger P, Meyerhardt J, Attawia M, Lawrence C \& Fuchs CA 2006 a A phase II study of temozolomide and bevacizumab in patients with advanced neuroendocrine tumors. Journal of Clinical Oncology 24 4044. (doi:10.1200/JCO.2006.06.9823)

Kulke MH, Stuart K, Enzinger PC, Ryan DP, Clark JW, Muzikansky A, Vincitore M, Michelini A \& Fuchs CS $2006 b$ Phase II study of temozolomide and thalidomide in patients with metastatic neuroendocrine tumors. Journal of Clinical Oncology 24 401-406. (doi:10.1200/ JCO.2005.03.6046)

Kulke M, Blaszkowsky L, Zhu A, Florio S, Regan E, Ryan D \& Chan J 2010 Phase I/II study of everolimus (RAD001) in combination with temozolomide (TMZ) in patients (pts) with advanced pancreatic neuroendocrine tumors (NET). In 2010 ASCO Gastrointestinal Cancers Symposium. Abstract 223.

Kulke MH, Siu LL, Tepper JE, Fisher G, Jaffe D, Haller DG, Ellis LM, Benedetti JK, Bergsland EK, Hobday TJ et al. 2011 Future directions in the treatment of neuroendocrine tumors: consensus report of the National Cancer Institute Neuroendocrine Tumor clinical trials planning meeting. Journal of Clinical Oncology 29 934-943. (doi:10.1200/ JCO.2010.33.2056)

Lam T, Hetherington JW, Greenman J, Little S \& Maraveyas A 2007 Metronomic chemotherapy dosingschedules with estramustine and temozolomide act synergistically with anti-VEGFR-2 antibody to cause inhibition of human umbilical venous endothelial cell growth. Acta Oncologica 46 1169-1177. (doi:10.1080/ 02841860701373603)

Modlin IM, Oberg K, Chung DC, Jensen RT, de Herder WW, Thakker RV, Caplin M, Delle Fave G, Kaltsas GA, Krenning EP et al. 2008 Gastroenteropancreatic neuroendocrine tumours. Lancet Oncology 9 61-72. (doi:10. 1016/S1470-2045(07)70410-2)

Oberg K 2002 Management of neuroendocrine tumors. In ASCO Annual Meeting, pp 401-405.

Strosberg JR, Fine RL, Choi J, Nasir A, Coppola D, Chen DT, Helm J \& Kvols L 2011 First-line chemotherapy with capecitabine and temozolomide in patients with metastatic pancreatic endocrine carcinomas. Cancer 117 268-275. (doi:10.1002/cncr.25425)

Tolcher AW, Gerson SL, Denis L, Geyer C, Hammond LA, Patnaik A, Goetz AD, Schwartz G, Edwards T, Reyderman L et al. 2003 Marked inactivation of O6alkylguanine-DNA alkyltransferase activity with protracted temozolomide schedules. British Journal of Cancer 88 1004-1011. (doi:10.1038/sj.bjc.6600827)

Welin S, Sorbye H, Sebjornsen S, Knappskog S, Busch C \& Oberg K 2011 Clinical effect of temozolomide-based chemotherapy in poorly differentiated endocrine carcinoma after progression on first-line chemotherapy. Cancer 117 4617-4622. (doi:10.1002/cncr.26124)

Yao JC, Phan A, Hoff PM, Chen HX, Charnsangavej C, Yeung SC, Hess K, Ng C, Abbruzzese JL \& Ajani JA 2008 Targeting vascular endothelial growth factor in advanced carcinoid tumor: a random assignment phase II study of depot octreotide with bevacizumab and pegylated interferon alpha-2b. Journal of Clinical Oncology 26 1316-1323. (doi:10.1200/JCO.2007.13. 6374) 